\title{
Octahedral tilting in Prussian blue analogues
}

\author{
Hanna L. B. Boström ${ }^{1}$, William R. Brant ${ }^{2}$, Anthony E. Phillips ${ }^{3}$ \\ ${ }^{1}$ Max Planck Institute for Solid State Research, Germany, ${ }^{2}$ Uppsala Universitet, Sweden, ${ }^{3}$ Queen Mary University of London, UK

\section{h.bostroem@fkf.mpg.de}

Octahedral tilting is integral to the structure and functionality of perovskites: tilt distortions influence the electronic and magnetic properties [1] and reduce the macroscopic symmetry, as rationalised by group theory [2]. Since tilts are driven by the relative sizes of the metal ions, compositional modification can allow for the control of tilt patterns to achieve desired functionality, such as multiferroicity [3]. A class of materials closely related to perovskites are the Prussian blue analogues (PBAs), where cyanide anions replace the oxides to give the formula $\mathrm{A}_{\mathrm{x}} \mathrm{M}\left[\mathrm{M}^{\prime}(\mathrm{CN})_{6}\right]_{1-y} \square_{\mathrm{y}} \cdot n \mathrm{H}_{2} \mathrm{O}$ (A is an alkali metal, $\mathrm{M}$ and $\mathrm{M}^{\prime}$ are transition metals and $\square$ denotes a vacancy). Like double perovskites, the parent structure (aristotype) adopts the space group $F m \overline{3} m$, although ordered A-site cations $(x>$ 1) or vacancies $(y>1)$ may reduce the symmetry to $F \overline{4} 3 m$ or $P m \overline{3} m$.

Due to the similarity to perovskites, octahedral tilting also features in PBAs and can have a strong impact on the functional response. To illustrate, the tilts in $\mathrm{Na}_{2} \mathrm{MnMn}(\mathrm{CN})_{6}$ nearly triples the magnetic ordering temperature compared to the cubic $\mathrm{Cs}_{2} \mathrm{MnMn}(\mathrm{CN})_{6}[4]$. However, the tilting in PBAs is poorly understood, which is evidenced by considerable confusion in the literature. A systematic understanding of the factors underlying octahedral tilting in PBAs would be beneficial and facilitate tilt engineering approaches.

Here, density functional theory (DFT) calculations and literature surveys are used to identify and rationalise the trends in octahedral tilting for PBAs. A high concentration of A-site cations is a prerequisite for tilting and PBAs with $x<1$ are almost invariably cubic, even upon cooling. Moreover, the A-site cation radius dictates the particular tilt pattern [Fig. 1], in line with the behaviour of perovskites. $\mathrm{M}^{\prime}(\mathrm{CN})_{6}$ vacancies - which have no analogue in oxide perovskites - do not appear to play a major role, but the presence of interstitial water stipulates which tilt pattern that appears in response to external or chemical pressure. Functional implications of the tilts include the tilt-driven improper ferroelectricity in the high-pressure $P n$ phase of $\mathrm{RbMnCo}(\mathrm{CN})_{6}$ [5], or the undesirable tilt transition upon Na intercalation in cathode materials based on PBAs [6]. More generally, our results help develop a unified picture of the structural behaviour of PBAs and also improve the understanding of tilting distortions in general.

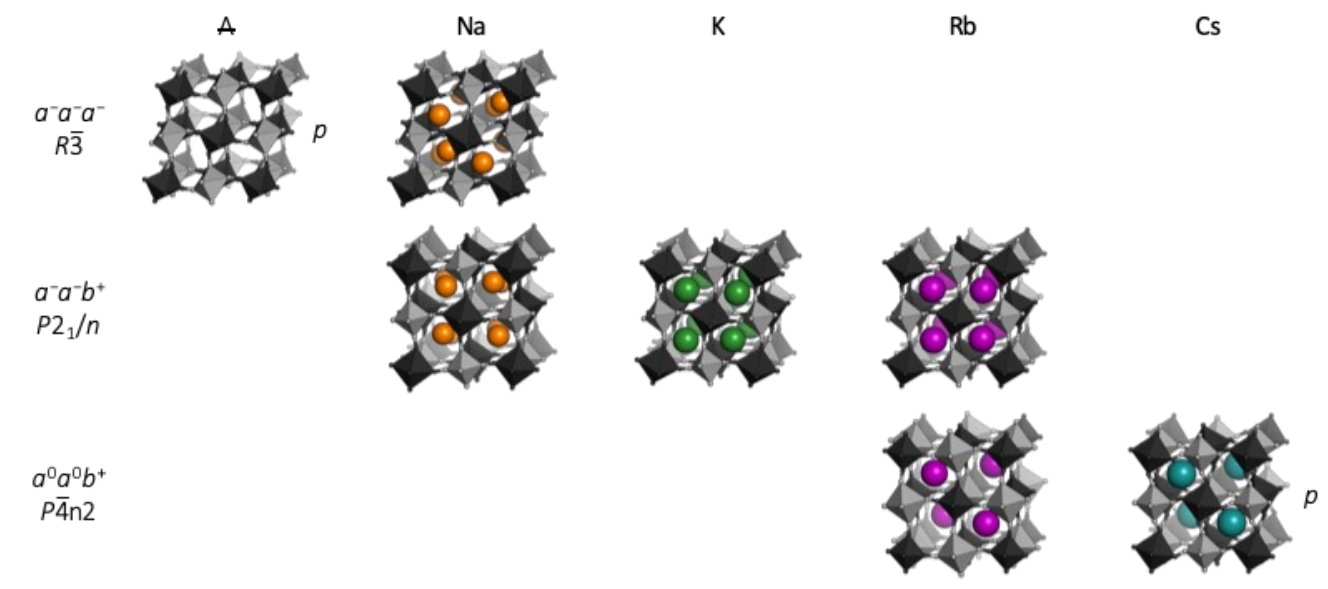

Figure 1. Tilt distortions in PBAs as a function of the A-site cation. Structures that only tilt under compression are marked with ' $p$ '.

[1] Bull, C. L., \& McMillan, P. F. (2004). J. Solid State Chem., 177, 2323.

[2] Howard, C. J., Kennedy, B. J., \& Woodward, P. M. (1999). Acta Cryst. B, 59, 463.

[3] Pitcher, M. J., Mandal, P., Dyer, M. S., Alaria, J., Borisov, P., Niu, H., Claridge, J. B. \& Rosseinsky, M. J. (2015). Science, 347, 420.

[4] Kareis, C. M., Lapidus, S. H., Her, J.-H., Stephens, P. W., \& Miller, J. S. (2012). J. Am. Chem. Soc., $134,2246$.

[5] Boström, H. L. B., Collings, I. E., Daisenberger, D., Ridley, C. J., Funnell, N. P., \& Cairns, A. B. (2021). J. Am. Chem. Soc., 143, 3544.

[6] Asakura, D., Okubo, M., Mizuno, Y., Kudo, T., Zhou, H., Ikedo, K., Mizokawa, T., Okazawa, A., \& Kojima, N. (2012). J. Phys. Chem. C, 116, 8364.

Keywords: Prussian blue analogues; perovskites; tilting; group theory

Acta Cryst. (2021), A77, C131 